\title{
Modelling Anisotropic Viscoelasticity for Real-Time Soft Tissue Simulation
}

\author{
Zeike A. Taylor ${ }^{1}$, Olivier Comas ${ }^{2}$, Mario Cheng ${ }^{2}$, Josh Passenger ${ }^{2}$, \\ David J. Hawkes ${ }^{1}$, David Atkinson ${ }^{1}$, and Sébastien Ourselin ${ }^{1}$ \\ ${ }^{1}$ Centre for Medical Image Computing, University College London, Gower St, \\ London, WC1E 6BT, UK \\ ${ }^{2}$ BioMedIA Lab, e-Health Research Centre, CSIRO ICT Centre, Level 20, 300 \\ Adelaide St, Brisbane, QLD, 4000, Australia \\ z.taylor@cs.ucl.ac.uk
}

\begin{abstract}
Previously almost all biomechanically-based time-critical surgical simulation has ignored the well established features of tissue mechanical response of anisotropy and time-dependence. We address this issue by presenting an efficient solution procedure for anisotropic viscohyperelastic constitutive models which allows use of these in nonlinear explicit dynamic finite element algorithms. We show that the procedure allows incorporation of both anisotropy and viscoelasticity for as little as $5.1 \%$ additional cost compared with the usual isotropic elastic models. When combined with high performance GPU execution the complete framework is suitable for time-critical simulation applications such as interactive surgical simulation and intraoperative image registration.
\end{abstract}

\section{Introduction}

In recent years there has been growing interest in the use of computational biomechanics as a basis for simulation of soft tissues deformation. Example applications include interactive simulation environments [1/2,3 and biomechanically driven image registration 456]. Both such applications may be subject to stringent solution time constraints; interactive simulation requires solutions to be obtained at visual, or even haptic feedback rates $(>500 \mathrm{~Hz})$, while intraoperative image registration must be fast enough that the work flow of the surgical procedure is not interrupted. Therefore viable simulation procedures for these applications are necessarily those that yield rapid solutions.

While progress has been made with respect to inclusion of constitutive and kinematic nonlinearities, virtually all previous work in this area has ignored the well established phenomena of anisotropy and time-dependence (predominantly manifested as stress relaxation, creep, hysteresis, and strain rate-dependence) of tissue mechanical response [7]. Notable exceptions include [28], in which liver and facial muscles were treated as transversely isotropic, and 9,10, in which viscoelastic effects were included. Of these, only [2] reported real-time solution speeds, and to the best of our knowledge no authors have included both 
anisotropy and viscoelasticity in time-critical applications. In light of the abundant experimental evidence testifying the importance of these phenomena [117] and the comprehensive modelling approaches developed for their analysis [12, the issue appears to be one of computational complexity and the mentioned time-constraints.

We address this issue by presenting a constitutive update procedure for viscohyperelastic materials suitable for use in explicit finite element algorithms. Material models of this type have been shown to very accurately reproduce the phenomena described above [117]. The formulation is general in the sense that any underlying elastic response may be included. In particular we show that kinematically consistent anisotropic formulations valid for large deformations are naturally accommodated. We demonstrate the validity and efficiency of the procedure within a graphics processor (GPU) -based finite element solver (specifically, a total Lagrangian explicit dynamic (TLED) solver [131415]), and show that the framework is suitable for use in time-critical applications.

\section{TLED Finite Element Algorithm}

A complete description of the TLED algorithm is available in previous publications 1315, where it has been shown to allow very rapid nonlinear analysis of soft tissues. Briefly, the algorithm consists of a precomputation phase in which various element and system quantities are calculated, followed by a time-loop in which incremental solutions for the node displacements $\mathbf{u}$ are found. During each step of the time-loop we

1. Apply loads (displacements and/or forces) and boundary conditions to relevant nodal degrees of freedom

2. For each element compute

(a) deformation gradient $\mathbf{F}$ and right Cauchy-Green deformation tensor $\mathbf{C}$

(b) linear strain-displacement matrix $\mathbf{B}_{L}$

(c) $2^{\text {nd }}$ Piola-Kirchhoff stress $\mathbf{S}$

(d) element nodal forces $\tilde{\mathbf{f}}$, and add these to the total nodal forces $\mathbf{f}$

3. For each node compute new displacements $\mathbf{u}$ using the central difference method.

For under-integrated 8-node hexahedral elements the nodal force contributions from each element are obtained (via Gaussian quadrature) from $\tilde{\mathbf{f}}=8 \mathbf{B}_{L}^{T} \check{\mathbf{S}}_{\operatorname{set}} \mathbf{J}$, where $\mathbf{J}$ is the precomputed element Jacobian matrix and $\check{\mathbf{S}}$ is the vector form of the stress tensor $\mathbf{S}$. This equation makes no assumption concerning the constitutive model employed. In the subsequent sections we describe a general formulation for anisotropic viscoelasticity, particular cases of which have been shown to be excellent models of the mechanical response of many soft tissues.

\section{Anisotropic Visco-hyperelastic Constitutive Equations}

In continuum mechanics the Helmholtz free energy (strain energy) function $\Psi$ encapsulates the energy per unit volume associated with deformation of a material, 
and is the standard representation of the material's constitutive response. For hyperelastic materials $\Psi$ is a function of the current deformation only, whereas for visco-hyperelastic materials it is a function of the entire deformation history. A complete visco-hyperelastic response may be obtained by augmenting hyperelastic strain energy functions with time dependent relaxation terms and expressing in convolution integral form. This affords generality since any underlying hyperelastic form may be used. In particular the formulation naturally accommodates anisotropic hyperelastic terms. We present equations for the case of transverse isotropy, which is the simplest class of anisotropy.

\subsection{Hyperelastic Response}

Transversely isotropic (TI) materials are characterised by a single preferred direction a; the mechanical response is isotropic in the plane orthogonal to a. In the present work we consider TI strain energy functions with separated isochoric (volume-preserving) and volumetric terms [12] $\Psi(\mathbf{C}, \mathbf{a})=\Psi^{\text {iso }}\left(\bar{I}_{1}, \bar{I}_{2}, \bar{I}_{4}, \bar{I}_{5}\right)+$ $\Psi^{\operatorname{vol}}(J)$, where $J=\operatorname{det} \mathbf{F}$. Here $\bar{I}_{1}=\operatorname{tr} \overline{\mathbf{C}}$ and $\bar{I}_{2}=\left[(\operatorname{tr} \overline{\mathbf{C}})^{2}-\operatorname{tr}\left(\overline{\mathbf{C}}^{2}\right)\right] / 2$ are the first two invariants of the modified right Cauchy-Green deformation tensor $\overline{\mathbf{C}}=J^{-2 / 3} \mathbf{F}^{T} \mathbf{F}$, while $\bar{I}_{4}=\mathbf{a} \cdot \overline{\mathbf{C}} \mathbf{a}$ and $\bar{I}_{5}=\mathbf{a} \cdot \overline{\mathbf{C}}^{2} \mathbf{a}$ are pseudo-invariants of both $\overline{\mathbf{C}}$ and a. It may be observed that $\bar{I}_{1,2}$ are functions of deformation $\overline{\mathbf{C}}$ only, and therefore produce isotropic strain energy terms. In contrast $\bar{I}_{4,5}$ are directiondependent, and produce strain energy terms associated with deformation in the direction a only - effectively producing a different stiffness in this direction.

The stresses are obtained by differentiation of $\Psi: \mathbf{S}=2 \partial_{\mathbf{C}} \Psi=\mathbf{S}^{\text {iso }}+\mathbf{S}^{\text {vol }}$, where $\partial_{x}$ denotes differentiation with respect to $x . \mathbf{S}^{\text {iso }}$ and $\mathbf{S}^{\text {vol }}$ are stresses obtained from differentiation of isochoric and volumetric strain energy terms, respectively, and may be shown to be 12

$$
\mathbf{S}^{\mathrm{vol}}=2 \partial_{\mathbf{C}} \Psi^{\mathrm{vol}}=J p \mathbf{C}^{-1}, \quad \mathbf{S}^{\text {iso }}=2 \partial_{\mathbf{C}} \Psi^{\text {iso }}=J^{-2 / 3} \operatorname{Dev} \overline{\mathbf{S}},
$$

where

$$
\overline{\mathbf{S}}=\bar{\gamma}_{1} \mathbf{I}+\bar{\gamma}_{2} \overline{\mathbf{C}}+\bar{\gamma}_{4} \mathbf{a} \otimes \mathbf{a}+\bar{\gamma}_{5}(\mathbf{a} \otimes \overline{\mathbf{C}} \mathbf{a}+\overline{\mathbf{C}} \mathbf{a} \otimes \mathbf{a}) .
$$

Here $\bar{\gamma}_{1}=2\left(\partial_{\bar{I}_{1}} \Psi^{\text {iso }}+\bar{I}_{1} \partial_{\bar{I}_{2}} \Psi^{\text {iso }}\right), \bar{\gamma}_{a}=-2 \partial_{\bar{I}_{a}} \Psi^{\text {iso }}(a=2,4,5), p=\mathrm{d} \Psi^{\text {vol }} / \mathrm{d} J$ is the hydrostatic pressure, and $\operatorname{Dev}(\bullet)=(\bullet)-(1 / 3)[(\bullet): \mathbf{C}] \mathbf{C}^{-1}$ is the referential configuration deviatoric operator for a second order tensor.

Eqns. (11) and (2) are the general form of a TI hyperelastic stress response, defined in terms of invariants. They represent a kinematically consistent framework valid for large deformations. Specification of particular forms for $\Psi^{\text {iso }}$ and $\Psi^{\mathrm{vol}}$ would be motivated by the particular tissue under analysis, and may stem from phenomenological or microstructural considerations.

\subsection{Viscoelastic Response}

As mentioned, viscoelastic energy functions $\hat{\Psi}(\mathbf{C}, \mathbf{a}, t)$ valid for large deformations may be formulated by including relaxation functions $\alpha(t)=1-\sum_{i=1}^{N} \alpha_{i}(1-$ $\left.\exp \left(-t / \tau_{i}\right)\right)$, where $N, \alpha_{i}$ and $\tau_{i}$ are constants, and $t$ is time, and expressing 
the strain energy function in convolution integral form: $\hat{\Psi}(\mathbf{C}, \mathbf{a}, t)=\int_{0}^{t} \alpha(t-$ $\tau) \partial_{\tau} \Psi(\mathbf{C}, \mathbf{a}) \mathrm{d} \tau$. Such a form views the stress response at any time $t$ as the sum of responses to excitations at all previous times - the mentioned deformation history-dependence. If separated isochoric and volumetric terms are used, as above, relaxation functions may be applied to either or both independently. Stresses are then obtained as

$$
\begin{aligned}
& \mathbf{S}^{\text {iso }}=2 \partial_{\mathbf{C}} \hat{\Psi}^{\text {iso }}=\int_{0}^{t} \alpha^{\text {iso }}(t-\tau) \partial_{\tau} \boldsymbol{\Phi}^{\text {iso }} \mathrm{d} \tau \\
& \mathbf{S}^{\mathrm{vol}}=2 \partial_{\mathbf{C}} \hat{\Psi}^{\mathrm{vol}}=\int_{0}^{t} \alpha^{\mathrm{vol}}(t-\tau) \partial_{\tau} \boldsymbol{\Phi}^{\mathrm{vol}} \mathrm{d} \tau
\end{aligned}
$$

where $\boldsymbol{\Phi}^{\text {iso }}=2 \partial_{\mathbf{C}} \Psi^{\text {iso }}$ and $\boldsymbol{\Phi}^{\mathrm{vol}}=2 \partial_{\mathbf{C}} \Psi^{\mathrm{vol}}$ are the instantaneous hyperelastic stress responses (Eqns. (11). Compared with use of purely hyperelastic models, in which stress may be computed directly from the (known) current deformation, use of visco-hyperelastic models within the TLED algorithm requires a constitutive update scheme involving time integration of the stress Eqns. (4).

\section{Constitutive Update Procedure for Explicit Analyses}

In the following we derive equations based on $\mathbf{S}^{\text {iso }}$, and note that an equivalent procedure may be applied for the volumetric term, if applicable. Referring to Eqn. (41) we note that $\mathbf{S}^{\text {iso }}$ may be restated as $\mathbf{S}^{\text {iso }}=\boldsymbol{\Phi}^{\text {iso }}-\sum_{i=1}^{N^{\text {iso }}} \boldsymbol{\Upsilon}_{i}^{\text {iso }}$, where

$$
\boldsymbol{\Upsilon}_{i}^{\text {iso }}=\int_{0}^{t} \alpha_{i}^{\text {iso }}\left(1-\exp \left((\tau-t) / \tau_{i}^{\text {iso }}\right)\right) \partial_{\tau} \boldsymbol{\Phi}^{\text {iso }} \mathrm{d} \tau, \quad i \in\left[1, N^{\text {iso }}\right]
$$

are rate-dependent terms associated with each term in the relaxation functions. In an incremental analysis we require the stress at the current increment given the deformation state and history of the material. Adding subscripts to indicate time increments the isochoric stress may be updated using

$$
\mathbf{S}_{n}^{\text {iso }}=\boldsymbol{\Phi}_{n}^{\text {iso }}-\sum_{i=1}^{N^{\text {iso }}}\left(\Upsilon_{i}^{\text {iso }}\right)_{n} .
$$

As mentioned, instantaneous terms $\boldsymbol{\Phi}_{n}^{\text {iso }}$ may be computed from the current deformation $\mathbf{C}_{n}$ (Eqn. (1) $)_{2}$ ). The main difficulty then is computation of the incremental rate-dependent terms $\left(\Upsilon_{i}^{\text {iso }}\right)_{n}$. Our strategy is to maintain each $\Upsilon_{i}^{\text {iso }}$ as a state variable to be updated at each increment also. For clarity hereafter we consider only a single Prony term, but note that more terms may be added without difficulty.

Our approach is to convert the integral equation (5) into a rate form which may then be numerically integrated to produce an incremental update formula for $\Upsilon^{\text {iso }}$. It may be shown that such a rate form is given by

$$
\dot{\boldsymbol{\Upsilon}}^{\text {iso }}=\frac{1}{\tau_{\text {iso }}^{\text {iso }}}\left(\alpha^{\text {iso }} \boldsymbol{\Phi}^{\text {iso }}-\boldsymbol{\Upsilon}^{\text {iso }}\right) \text {. }
$$


Integration of (7) using the backward Euler method yields a formula for $\Upsilon_{n}^{\text {iso }}$ :

$$
\Upsilon_{n}^{\text {iso }}=A \boldsymbol{\Phi}_{n}^{\text {iso }}+B \Upsilon_{n-1}^{\text {iso }},
$$

where $A=\Delta t \alpha^{\text {iso }} /\left(\Delta t+\tau^{\text {iso }}\right)$ and $B=\tau^{\text {iso }} /\left(\Delta t+\tau^{\text {iso }}\right)$ are constant coefficients, and $\Delta t$ is the time step size. A similar expression may be obtained for volumetric terms.

The constitutive update procedure thus consists of (a) updating state variables (one for each Prony term) via Eqn. (8), and (b) updating stresses via Eqn. (6). We note in particular that when used with reduced integration hexahedra or linear tetrahedra the present scheme introduces only $12 N_{P}\left(N_{P}=N^{\text {iso }}+N^{\text {vol }}\right)$ extra multiplications per element per time step compared with an equivalent hyperelastic formulation. Additional storage requirements are also minimal since only state variables (6-vectors) are retained between increments.

\section{Algorithm Performance}

The procedure was implemented (within the TLED algorithm) for GPU execution using the CUDA API [16], however the arrangement of kernel computations was essentially the same as that of our earlier OpenGL-based implementation 15. We used reduced integration 8-node hexahedral elements which are preferable to the 4-node tetrahedra used in our previous implementation [15], both in terms of solution accuracy and computational efficiency.

We present examples based on compression and shear of cube models to demonstrate the validity and performance of the constitutive update procedure. We emphasise that these geometrically simple examples are aimed at verifying the numerical solution method (being the contribution of this paper), not at validating any particular constitutive model itself. In each case we used a TI visco-hyperelastic model with elastic strain energy components defined by

$$
\Psi^{\text {iso }}=\frac{\mu}{2}\left(\bar{I}_{1}-3\right)+\frac{\eta}{2}\left(\bar{I}_{4}-1\right)^{2}, \quad \Psi^{\mathrm{vol}}=\frac{\kappa}{2}(J-1)^{2},
$$

where $\mu$ is the small strain shear modulus, $\kappa$ is the bulk modulus, and $\eta$ is a material parameter with units of $\mathrm{Pa} . \bar{I}_{5}$-dependent terms were omitted for simplicity. We used viscoelastic isochoric terms only, with $N^{\text {iso }}=1$. Material parameters $\mu=6568 \mathrm{~Pa}, \kappa=326210 \mathrm{~Pa}, \alpha_{1}=0.5$, and $\tau_{1}=0.58 \mathrm{~s}$ were chosen based on recent results for the viscoelastic response of human liver in vivo [17]. In the absence of appropriate experimental data for liver we selected $\eta=2 \mu$. Since $\eta$ and $\mu$ are similarly dimensioned parameters, this ensures $\eta$ is of an appropriate order of magnitude.

We first assessed the effects of anisotropy on the deformation response. A cube model with preferred direction $\mathbf{a}=\left[0 \frac{1}{\sqrt{2}} \frac{1}{\sqrt{2}}\right]$ was compressed by $30 \%$ along the $x$-axis. The deformed shape is shown in Fig. 1 along with that of an isotropic

${ }^{1}$ In [17] an estimate of $\mu=19704 \mathrm{~Pa}$ is given, but it is also noted that the parenchyma itself is likely to be up to $3 \times$ less stiff. 


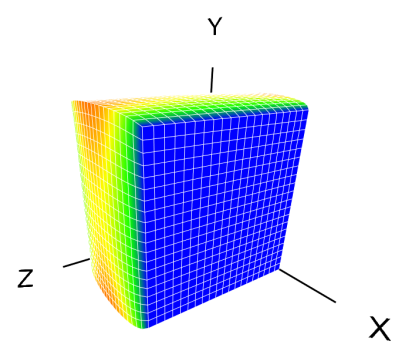

(a)

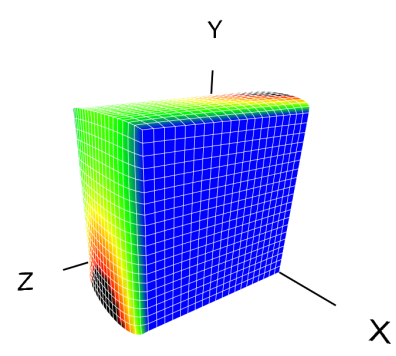

(b)

Fig. 1. Deformation pattern of (a) an isotropic model compared with (b) that of a TI model. Colour maps indicate magnitude of lateral displacement $\left(\left(u_{y}^{2}+u_{z}^{2}\right)^{1 / 2}\right)$

model (with $\eta=0$ ). With no preferred direction the expansion of the middle section was uniform (Fig. 1(a)). For the anisotropic model the direction defined by $y=z$ was stiffened, and Fig. 1(b) shows the resulting reduced expansion along this axis and the increased orthogonal expansion.

Next we confirmed the validity of the solution procedure by modelling a pure shear loading on the cube. Under such conditions an analytical solution for the stresses is available (omitted for brevity) and is compared with the numerical solutions in Fig. 2(a) for various loading speeds. The strain rate-dependence introduced by the model (and commonly observed in biological tissues) is clearly shown. Additionally the close match between the analytic and numerical solutions demonstrates the validity of the developed constitutive update scheme.

Finally we assessed the computational efficiency of the constitutive update scheme by measuring computation times for various constitutive models over a range of mesh densities. Using the cube geometry as above we generated models with mesh sizes between 3993 DOF and 177957 DOF. Three constitutive models were considered: the TI viscoelastic model (TIV), TIV minus the viscoelastic terms (TIE), and TIE minus the anisotropy terms (NHE - since this represents a neo-Hookean model). The test machine included an Intel Core2Duo $2.4 \mathrm{GHz}$ CPU, 2GB RAM, and an NVIDIA GeForce 8800GTX GPU.

Fig. 2(b) indicates that solution times are little affected by the introduction of the more complex constitutive models, and importantly by the use of the developed constitutive update scheme. We observe that the maximum solution time ratio for model TIE to model NHE (anisotropic vs isotropic) was 1.013, and that of model TIV to model TIE (viscoelastic vs elastic) was 1.043. The largest total solution time increase for an anisotropic viscoelastic model compared with an isotropic elastic one was 5.1\%. We conclude that the key features of anisotropy and viscoelasticity may be included in simulations at very little additional computational cost. Moreover, using the present material parameters 


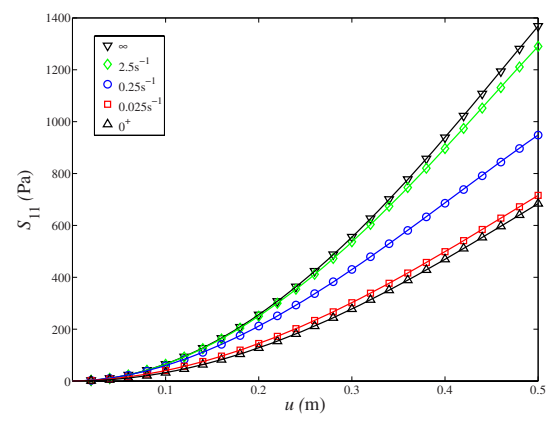

(a)

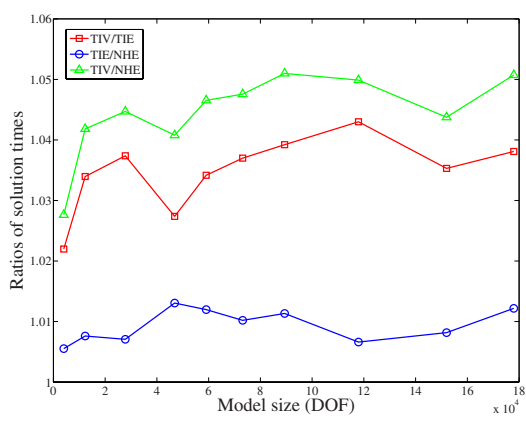

(b)

Fig. 2. (a) $S_{11}$ curves for pure shear deformation at varying strain rates. Curves for strain rates of $2.5 \mathrm{~s}^{-1}, 0.25 \mathrm{~s}^{-1}$, and $0.025 \mathrm{~s}^{-1}$ are given, along with the bounding instantaneous and equilibrium responses, labelled $\infty$ and $0^{+}$, respectively. Solid lines correspond to analytical solutions, while markers indicate the numerical solution. (b) ratios of solution times for the constitutive models: TIV to TIE, TIE to NHE, and TIV to NHE.

and GPU implementation models of up to approx. 10000 DOF may be solved in real-time. For more compliant tissues such as brain (for which the critical solution time step is larger) models of up to 55000 DOF may be solved in realtime.

\section{Conclusion}

There is abundant evidence that most biological tissues exhibit time-dependent mechanical responses, and that even non-load bearing organs exhibit directiondependence. These phenomena may be modelled using viscoelastic and anisotropic constitutive formulations. We presented a constitutive update scheme which allows use of such models within explicit finite element procedures for as little as $5.1 \%$ additional computational cost compared with isotropic elastic models. The scheme was general in the sense that any strain energy function (e.g. specific to particular organs) may be incorporated. Combined with GPU execution the presented scheme is suitable for real-time applications.

Explicit procedures have recently been shown to allow very rapid soft tissue simulation whilst retaining physically consistent kinematic nonlinearities. The present contribution expands the utility of such procedures and should find applications in time-critical simulation applications.

\section{Acknowledgements}

The financial support of the EPSRC (Grant reference: EP/F01144X/1) and the CSIRO Preventative Health Flagship is gratefully acknowledged. 


\section{References}

1. Cotin, S., Delingette, H., Ayache, N.: Real-time elastic deformations of soft tissues for surgery simulation. IEEE Transactions On Visualization and Computer Graphics 5(1), 62-73 (1999)

2. Picinbono, G., Delingette, H., Ayache, N.: Non-linear anisotropic elasticity for realtime surgery simulation. Graphical Models 65, 305-321 (2003)

3. Szekely, G., Brechbühler, C., Hutter, R., Rhomberg, A., Ironmonger, N., Schmid, P.: Modelling of soft tissue simulation for laparscopic surgery simulation. Medical Image Analysis 4, 57-66 (2000)

4. Clatz, O., Delingette, H., Talos, I.F., Golby, A.J., Kikinis, R., Jolesz, F.A., Ayache, N., Warfield, S.K.: Robust nonrigid registration to capture brain shift from intraoperative MRI. IEEE Transactions on Medical Imaging 24(11), 1417-1427 (2005)

5. Ferrant, M., Warfield, S.K., Nabavi, A., Macq, B., Kikinis, R.: Registration of 3D intraoperative MR images of the brain using a finite element biomechanical model. In: 3rd International Conference on Medical Image Computing and Computer Assisted Intervention, Pittsburgh, USA, pp. 19-28 (2000)

6. Miga, M.I., Paulsen, K.D., Hoopes, P.J., Kennedy Jr., F.E., Hartov, A., Roberts, D.W.: In vivo quantification of a homogeneous brain deformation model for updating preoperative images during surgery. IEEE Transactions on Biomedical Engineering 47(2), 266-273 (2000)

7. Humphrey, J.D.: Continuum biomechanics of soft biological tissues. Proceedings Of The Royal Society Of London Series A-Mathematical, Physical and Engineering Sciences 459, 3-46 (2003)

8. Chabanas, M., Luboz, V., Payan, Y.: Patient specific finite element model of the face soft tissues for computer-assisted maxillofacial surgery. Medical Image Analysis 7(2), 131-151 (2003)

9. Hu, J., Jin, X., Lee, J.B., Zhang, L., Chaudhary, V., Guthikonda, M., Yang, K.H., King, A.I.: Intraoperative brain shift prediction using a 3D inhomogeneous patientspecific finite element model. Journal of Neurosurgery 106(1), 164-169 (2007)

10. Wittek, A., Miller, K., Kikinis, R., Warfield, S.K.: Patient-specific model of brain deformation: Application to medical image registration. Journal of Biomechanics 40, 919-929 (2007)

11. Fung, Y.C.: Biomechanics: mechanical properties of living tissues, 2nd edn. Springer, New York (1993)

12. Holzapfel, G.A.: Nonlinear Solid Mechanics: A Continuum Approach for Engineering. John Wiley \& Sons, Chichester (2000)

13. Miller, K., Joldes, G., Lance, D., Wittek, A.: Total Lagrangian explicit dynamics finite element algorithm for computing soft tissue deformation. Communications in Numerical Methods in Engineering 23(2), 121-134 (2007)

14. Taylor, Z.A., Cheng, M., Ourselin, S.: Real-time nonlinear finite element analysis for surgical simulation using graphics processing units. In: 10th International Conference on Medical Image Computing and Computer Assisted Intervention, Brisbane, Australia, pp. 701-708 (2007)

15. Taylor, Z.A., Cheng, M., Ourselin, S.: High-speed nonlinear finite element analysis for surgical simulation using graphics processing units. IEEE Transactions on Medical Imaging 27(5), 650-663 (2008)

16. NVIDIA Corporation: NVIDIA CUDA Programming Guide Version 1.1 (2007)

17. Nava, A., Mazza, E., Furrer, M., Villiger, P., Reinhart, W.H.: In vivo mechanical characterization of human liver. Medical Image Analysis 12(2), 203-216 (2008) 